\title{
Injectable Alginate Hydrogel Cross-Linked by Calcium Gluconate- Loaded Porous Microspheres for Cartilage Tissue Engineering
}

\author{
JinFeng Liao,,$^{\dagger}$ BeiYu Wang, ${ }^{\dagger}$ YiXing Huang, $"$ Ying Qu, ${ }^{\dagger}$ JinRong Peng, ${ }^{\dagger}$ and ZhiYong Qian*, ${ }^{\dagger}$ \\ ${ }^{\dagger}$ State Key Laboratory of Biotherapy and Cancer Center, and Collaborative Innovation Center for Biotherapy, West China Hospital \\ and ${ }^{\ddagger}$ State Key Laboratory of Oral Diseases, National Clinical Research Center for Oral Diseases, West China Hospital of \\ Stomatology, Sichuan University, Chengdu 610041, P. R. China \\ "Department of Orthopaedic Surgery, Second Affiliated Hospital of Wenzhou Medical University, Wenzhou 325027, P. R. China
}

\section{Supporting Information}

\begin{abstract}
A great interest has been shown in the injectable scaffolds for cartilage tissue regeneration because it can fill irregularly shaped defects easily through minimally invasive surgical treatments. Herein, we developed a new injectable three-dimensional (3D) alginate hydrogel loaded with biodegradable porous poly $(\varepsilon$-caprolactone $)-b$-poly(ethylene glycol) $-b$-poly $(\varepsilon$-caprolactone) microspheres (MPs/Alg) as the calcium gluconate container to cross-link alginate. Suspensions of chondrocytes/alginate and porous microspheres turned into a gel because of the release of calcium gluconate; thus, the injectable composite hydrogels

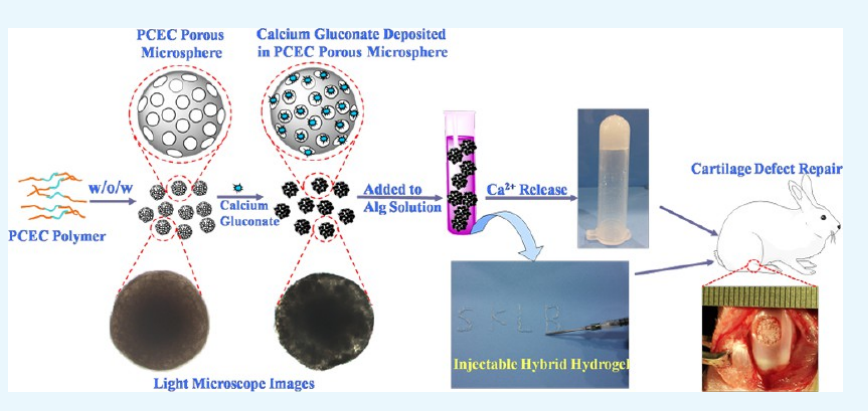
give a $3 \mathrm{D}$ scaffold to fit the defects perfectly and integrate the extracellular-matrix-mimicking architecture to efficiently accommodate cartilage cells in situ. Tissue repair in a full-thickness cartilage defect model was controlled at 6,12 , and 18 weeks after the implant by micro-CT and immunohistochemistry to evaluate the healing status. The defect in the MPs/Alg+ cells group achieved an almost complete repair at 18 weeks, and the repaired chondrocytes regained a normal tissue structure. Moreover, the MPs/Alg+ cells-treated group increased the quality of tissue formed, including the accumulated glycosaminoglycan and the uniformly deposited type II collagen. The results point out the promising application of the injectable MPs/Alg-chondrocytes system for cartilage tissue engineering.
\end{abstract}

\section{INTRODUCTION}

The self-repairing ability of articular cartilage after damage is very limited because of the low metabolic and biosynthetic activities of mature chondrocytes and the nonavailability of chondrogenic cells. ${ }^{1-3}$ Many strategies have been applied to enhance the cartilage defect repair with the ultimate aim of filling the defects with the same morphological and functional repaired cartilage tissue. Osteochondral allografting, periosteal and perichondral tissue grafting, chondrogenic cell transplantation, and subchondral drilling have been widely employed in preclinic and clinic trials. ${ }^{4}$ The concept of tissue engineering has been used to develop cell-based repair biomaterials. ${ }^{5-8}$ Numerous studies using chondrocytes or cells with chondrogenic potential (mesenchymal stem cells and adipose stem cells) have suggested that different biomaterials can support the proteoglycan-containing tissues and the formation of type II collagen (COL II) ${ }^{9,10}$ Specific extracellular matrix components of the articular cartilage, such as glycosaminoglycan (GAG) and collagen, play critical roles in supporting chondrogenesis and regulating the expression of the chondrocytic phenotype. ${ }^{11,12}$

Injectable scaffolds have been paid particular attention for cartilage tissue engineering because of their ability to fill irregularly shaped defects by minimally invasive procedures. The injectable tissue engineering system has applications in numerous materials. The scaffold of an injectable tissue engineering system must possess physical properties that allow it to be injected via a syringe or catheter. ${ }^{13-15}$ However, when implanted in the body, the scaffold materials should maintain a desired form or shape in defect location without diffusion or movement, acquiring more significant mechanical properties. ${ }^{16}$ Hydrogels are a class of materials that meet the requirements for a successful injectable tissue engineering system. Many methods have been employed to prepare injectable in situ forming hydrogels, including photopolymerization, ${ }^{17,18}$ enzymatic cross-linking, ${ }^{19,20}$ and chemical crosslinking with cross-linker agents (such as glutaraldehyde, carbodiimide, genipin, adipic dihydrazide, etc.). ${ }^{21-24}$ Unfortunately, photopolymerization usually needs a photosensitizer and prolonged irradiation, which limit its applications. On the other hand, toxic chemical cross-linkers are the major obstacles for chemical cross-linking to the use of injectable in situ forming scaffolds. ${ }^{25}$ It is worth mentioning that alginate is most commonly used as an instant hydrogel for bone tissue engineering because of its hydrophilicity, biocompatibility,

Received: December 11, 2016

Accepted: January 30, 2017

Published: February 9, 2017 


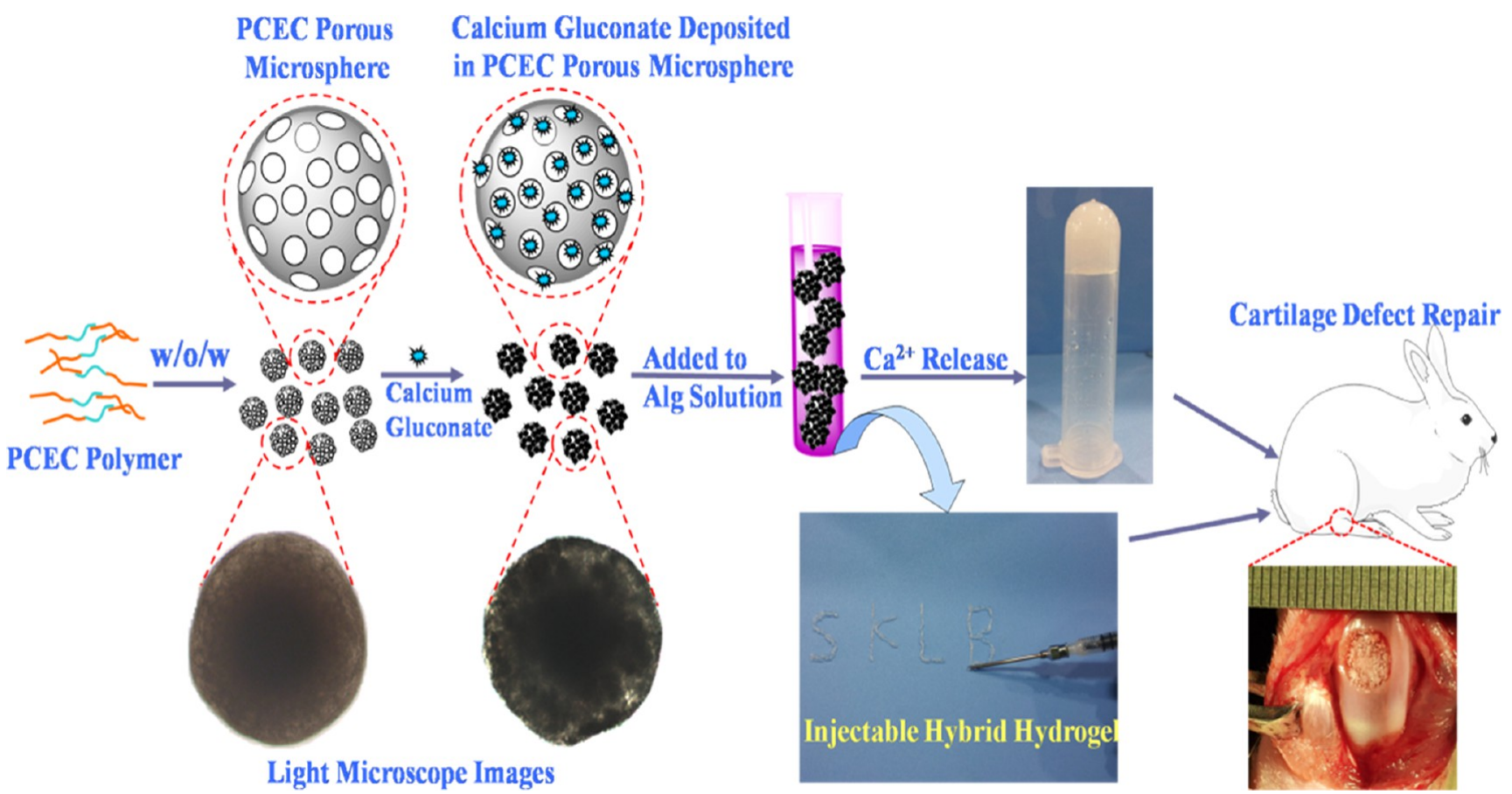

Figure 1. Preparation scheme of calcium gluconate loaded in porous PCEC microspheres/alginate hybrid hydrogel in situ formed in cartilage defect repair.

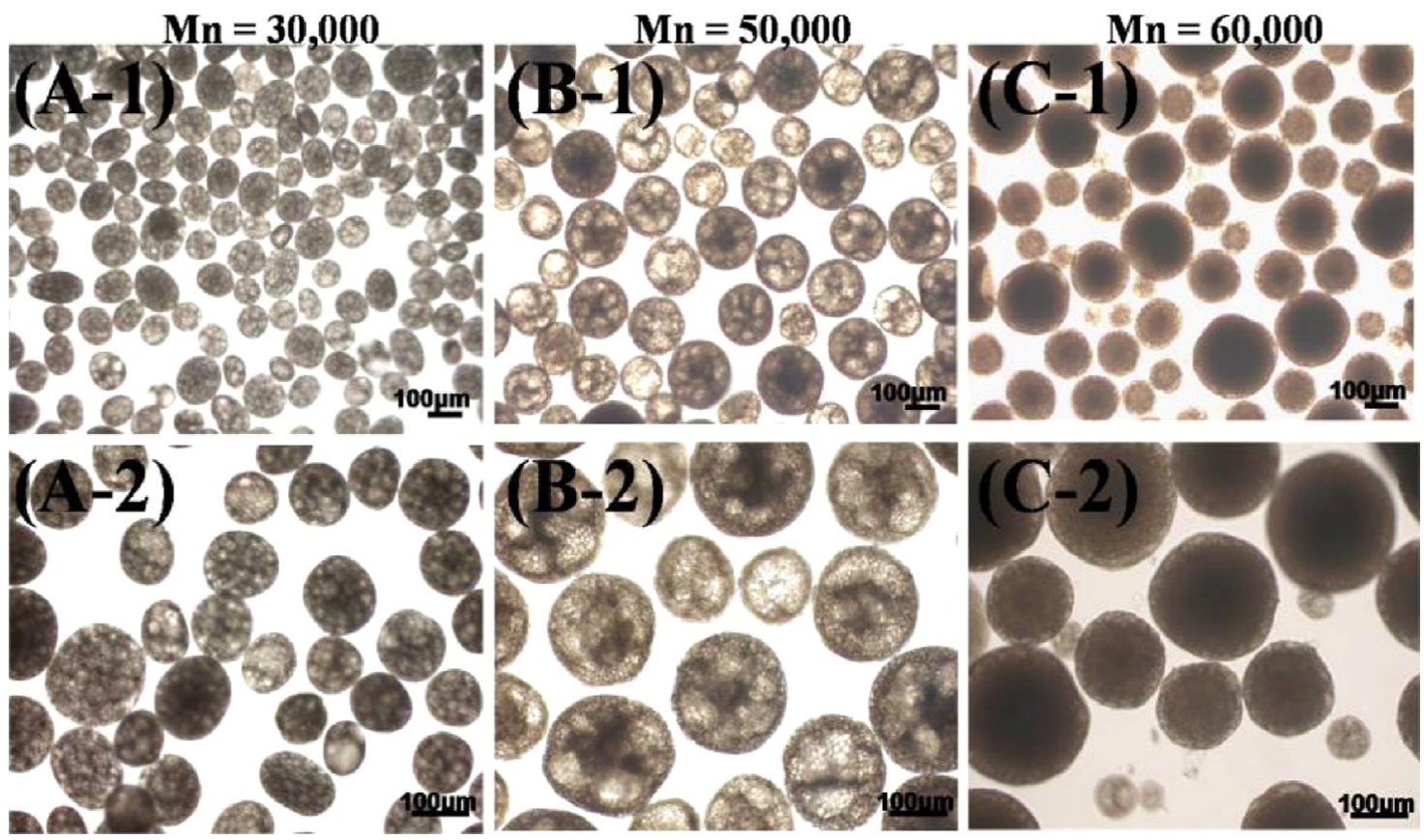

Figure 2. (A-1-C-1) Light microscope images of the porous PCEC microspheres made from PCEC copolymer with different molecular weights. A$1, \mathrm{~B}-1$, and $\mathrm{C}-1$ are the magnifications of A-1, B-1, and C-1, respectively.

and biodegradability. ${ }^{26,27}$ Alginate hydrogel with different shapes can be prepared by cross-linking at normal temperature in the absence of organic solvents. Chondrocytes encapsulated within calcium alginate hydrogels have shown a great promise as engineered scaffolds to repair articular cartilage, with the chondrocytes secreting cartilage markers, such as GAGs and COL II. ${ }^{28}$

On the other hand, most injectable hydrogels have limitations such as having sufficient mechanical stability and durability to support cell proliferation/differentiation before the formation of new tissue. One of the attempts to overcome this challenge was to use biodegradable microparticles, which have also been applied as injectable matrices for tissue regeneration and cell delivery. ${ }^{29}$ Mixtures of hydrogel and microspheres were widely used in cartilage repair; ${ }^{30}$ however, a simple mixing of these results in ignoring some properties like strength moduli and environmental responsibility.

Porous biodegradable microparticles are viewed as a useful tool for the delivery of proteins, ${ }^{31}$ antitumor drugs, ${ }^{32}$ and temporal templates for various tissue regeneration applica- 


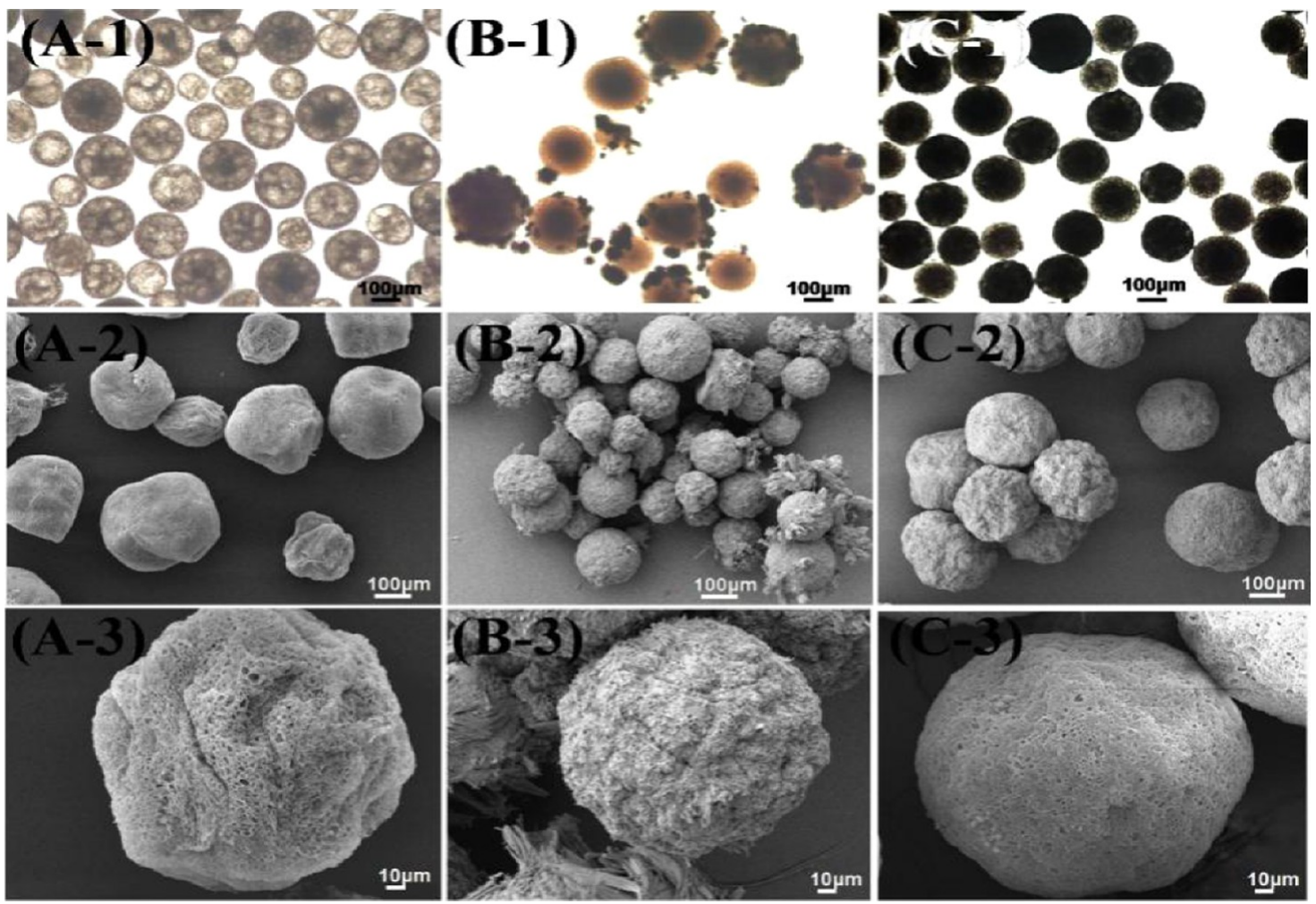

Figure 3. Microscope and SEM images of (A-1-A-3) porous PCEC microspheres; (B-1-B-3) porous PCEC microspheres loaded with calcium gluconate on the surface; and (C-1-C-3) porous PCEC microspheres loaded with calcium gluconate in the holes.

tions. $^{33,34}$ Porous microparticles made from block polymers, such as poly(D,L-lactic acid) (PDLLA) and poly(D,L-lactic-coglycolic acid) (PLGA), were generally prepared by a water-inoil-in-water $(\mathrm{w} / \mathrm{o} / \mathrm{w})$ double-emulsion solvent evaporation technique in tissue engineering. ${ }^{35-37}$ However, PDLLA and PLGA had some disadvantages, including the possibility of acute or chronic inflammatory responses, potential localized $\mathrm{pH}$ decreases because of the relative-acidity-degraded byproducts, and a retarded clearance rate. ${ }^{38,39}$ To overcome these limitations, amphiphilic poly $(\varepsilon$-caprolactone $)-b$-poly(ethylene glycol)- $b$-poly( $\varepsilon$-caprolactone) (PCL-PEG-PCL, abbreviated to PCEC) polymers were synthesized in our study and applied to prepare porous microspheres, which were loaded with calcium gluconate crystals as the cross-linker to cross-link alginate. The microspheres can release calcium gluconate once combined with an alginate solution. In this respect, we developed a novel hybrid injectable alginate hydrogel with porous PCEC microspheres as a container of calcium gluconate (MPs) and used the respective hydrogels as models for threedimensional (3D) scaffolds for in vitro and in vivo experiments. The suspension of the calcium gluconate/alginate mixture loaded with porous PCEC microspheres can be injected into a cartilage defect site and cross-linked in situ under normal physiological conditions for about $3 \mathrm{~min}$ with the release of calcium ions, thereby eliminating the need for invasive implantation procedures and retrieval surgeries (the preparation procedure of the hybrid hydrogel and cartilage defect repair in rabbit is shown in Figure 1). More specifically, the mechanical strength and the degradation rate of the scaffold may be controlled by the incorporation of porous PCEC microspheres. Furthermore, calcium gluconate may further enhance the cartilage tissue repair with calcium supply. ${ }^{40,41}$ This injectable hybrid hydrogel may yield synergetic effects, such as good mechanical strength, shape-persistent ability, stabilization of the microspheres, and biological performance. We would expect that this hybrid scaffold has a special advantage in cartilage and bone engineering. Furthermore, this is an unexplored biomaterial system so far to the best of our knowledge.

\section{RESULTS AND DISCUSSION}

2.1. Preparation and Characterization of Porous PCEC Microspheres. The PCEC copolymer was synthesized by ring-opening polymerization of $\varepsilon$-CL and PEG 4000. PCEC was successfully synthesized by characterization with ${ }^{1} \mathrm{H}$ NMR, as shown in Figure S1. The porous PCEC microspheres were prepared by a double-emulsification/solvent evaporation method with ammonium bicarbonate as the porogen. We tuned the molecular weight of the PCEC polymers to control their structural and morphological characteristics. As shown in Figure 2, for 30,50 , and $60 \mathrm{kDa}$ PCEC polymers, the average diameters of the microspheres were $124 \pm 23,203 \pm 35$, and $218 \pm 40 \mu \mathrm{m}$, respectively. The porous structure is homogeneous, and the pores were interconnected. With increasing molecular weight, the microspheres became spherical, and a more porous structure could be obtained. On the other hand, when the molecular weight of the polymer increased, the pores become smaller (pore sizes of microspheres made of 30,50 , and $60 \mathrm{kDa}$ were $\sim 10, \sim 8$, and $\sim 7 \mu \mathrm{m}$, respectively). Considering the more optimized morphology for cell growth, we chose the polymer with a molecular weight of $50 \mathrm{kDa}$ to prepare porous microspheres.

Next, scanning electron microscopy (SEM) of the microspheres $(50 \mathrm{kDa})$ was observed, as shown in Figure 3A-2,A-3. The average diameter of the open pore was about $8 \mu \mathrm{m}$ in the dry state. The pores may be larger than $8 \mu \mathrm{m}$ in the wet state, 

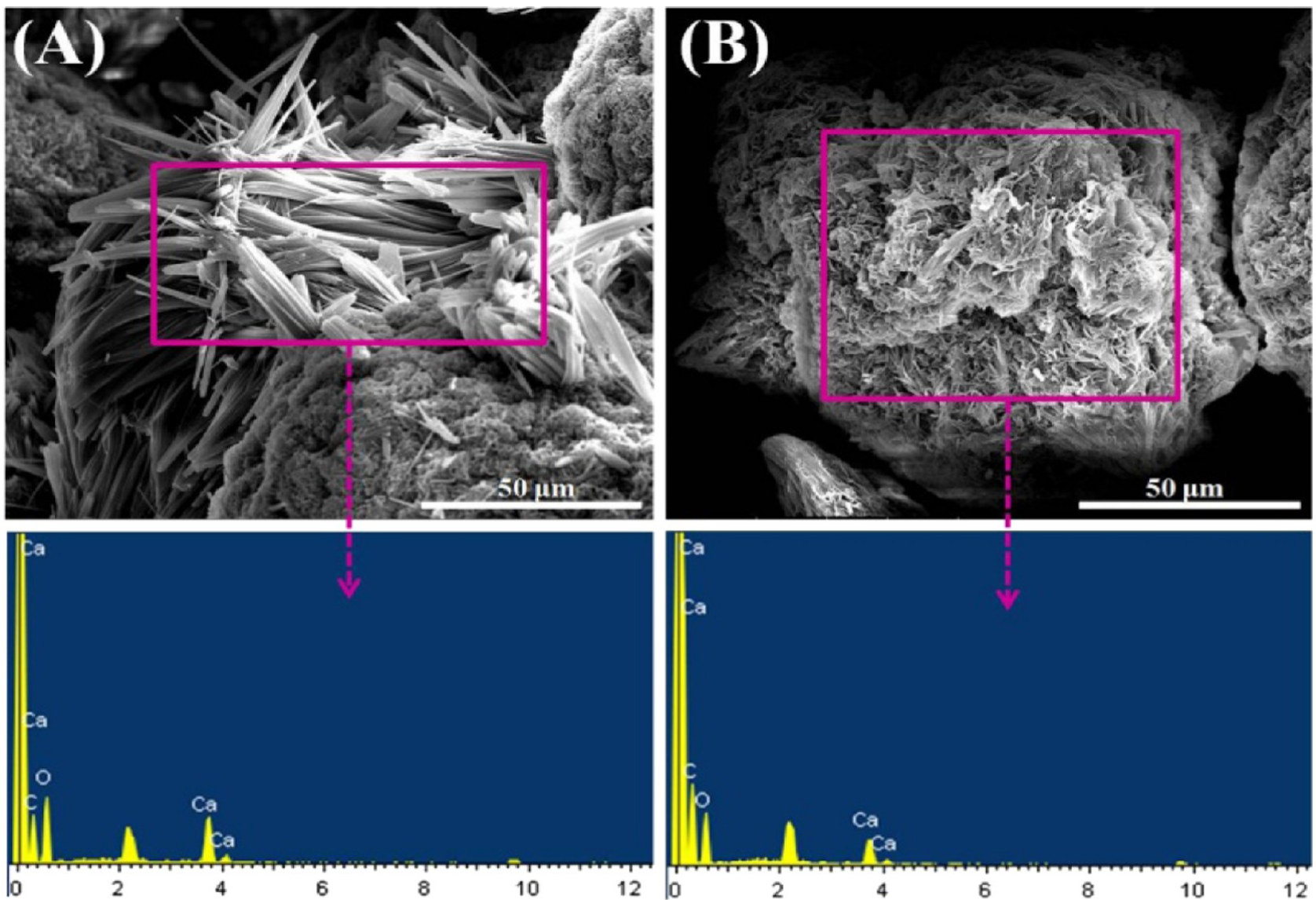

\begin{tabular}{lcc}
\hline Element & Weight Percentage & Atom Percentage \\
\hline C & $34.92 \%$ & $44.08 \%$ \\
O & $54.99 \%$ & $52.10 \%$ \\
Ca & $10.09 \%$ & $3.81 \%$ \\
\hline
\end{tabular}

\begin{tabular}{ccc}
\hline Element & Weight Percentage & Atom Percentage \\
\hline $\mathrm{C}$ & $50.68 \%$ & $59.45 \%$ \\
$\mathrm{O}$ & $43.86 \%$ & $38.63 \%$ \\
$\mathrm{Ca}$ & $5.46 \%$ & $1.92 \%$ \\
\hline
\end{tabular}

Figure 4. EDX element analysis of calcium gluconate crystal (A) on the surface and (B) in the holes of porous PCEC microspheres.

which was suitable for cell growth in the inner sides of the microspheres (Figure 3A-1). The wrinkling of the microspheres in the dry state is due to their hydrophilicity (amphiphilicity the PCEC polymer). The porosity of the PCEC microspheres was greater than $90 \%$, indicating a highly interconnected porous structure.

2.2. Porous PCEC Microspheres as the Container of Calcium Gluconate. Considering the insolubility of calcium gluconate in $\mathrm{EtOH}$, we have investigated the preparation method of calcium gluconate deposited in porous PCEC microspheres. Porous PCEC microspheres were first soaked in $\mathrm{EtOH}$, and a $3 \%$ calcium gluconate solution was added. This process was performed three times. Calcium gluconate deposited on the surface of the porous PCEC microspheres was observed by upright microscope and SEM (Figure 3B-1-B3). Energy-dispersive X-ray spectroscopy (EDX) scans obtained from the loaded calcium gluconate crystals showed that the weight percentage of calcium is $10.09 \%$ (Figure 4A). In another situation, porous PCEC microspheres were soaked in a $3 \%$ calcium gluconate solution first. Then, the calcium gluconate solution was decanted, and $\mathrm{EtOH}$ was added to deposit the calcium gluconate particles in the pores of the microspheres. This process was also performed three times. The holes of the porous PCEC microspheres were loaded with calcium gluconate, thereby decreasing the transmittance of light (Figure $3 \mathrm{C}-1$ ), and the microspheres became satiated (Figure 3C-2,C3 ). EDX analysis suggested that a trace amount of calcium element was deposited on the surface of the microspheres (Figure S2). To demonstrate calcium gluconate in the holes, we used liquid nitrogen to facilitate cryo-fracturing of the microspheres. As shown in Figure 4B, the broken microspheres were rough, and the calcium gluconate crystals were in the holes of the microspheres. EDX scans demonstrated that calcium gluconate was successfully loaded in the interior of the porous microspheres. The percentage of calcium in the holes was lower than that on the surface because of the interference of microspheres. Furthermore, to quantify the calcium content, the microspheres were soaked in water to release the whole loaded calcium ions. The amounts of calcium gluconate deposited on the surface or inner holes of porous PCEC microspheres measured by inductively coupled plasma-atomic emission spectrometer (ICP-AES) were 96 and $183 \mu \mathrm{g} / \mathrm{mg}$, respectively. Considering that the calcium gluconate crystals loaded on the surface of the microspheres tend to fall off and the time to form hydrogel when mixed with an alginate solution was short ( $\sim 30 \mathrm{~s})$, we chose the other one (loaded in the inner pores of the microspheres) for further study. 

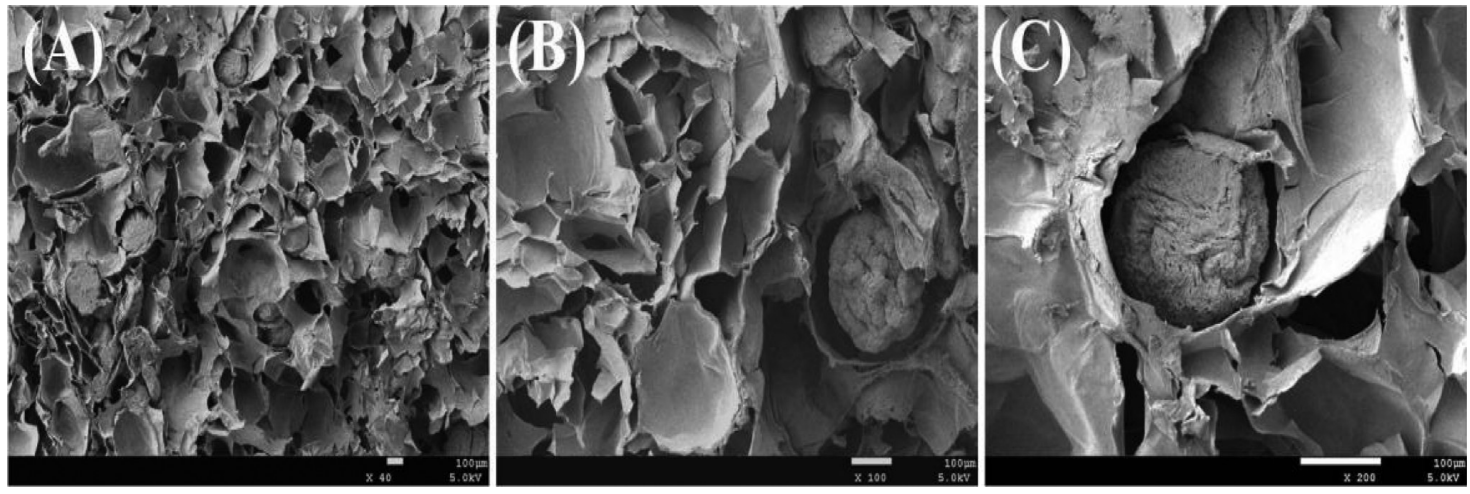

Figure 5. (A) SEM images and (B, C) the magnification of the cross section of porous PCEC microspheres/alginate hydrogels (scale bars were 100 $\mu \mathrm{m})$.
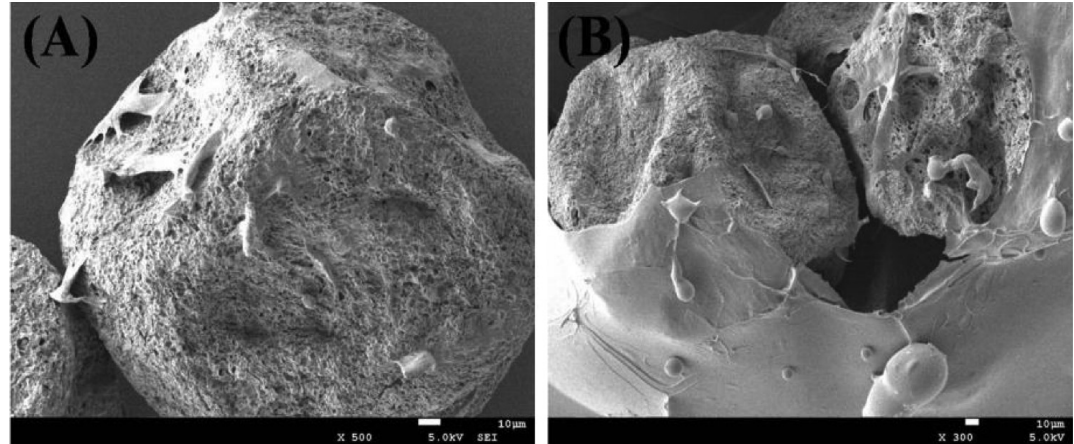

(C)

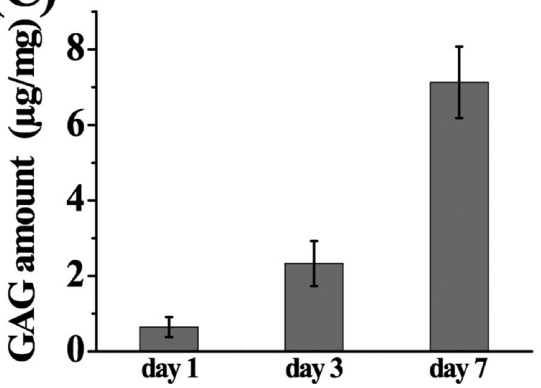

Figure 6. SEM photographs of (A) porous PCEC microspheres and (B) porous PCEC microspheres/alginate hydrogel cultured with cartilage cells for 3 days (scale bars were $10 \mu \mathrm{m}$ ); (C) GAG quantification assays after 1, 3, and 7 days of culture with cartilage cells on the microspheres/alginate hydrogel.

2.3. Characterization of Porous PCEC Microspheres/ Alginate Hybrid Hydrogel. Calcium gluconate crystals loaded in the pores of the microspheres $(50 \mathrm{mg})$ were mixed with a $1.5 \%$ alginate solution $(0.75 \mathrm{~mL})$. After homogenizing the mixture, these crystals can release calcium ions when contacting with water to cross-link alginate. The gel formation time was only $\sim 3 \mathrm{~min}$, leaving enough time to conduct the in vitro and in vivo experiments. Figure 5 displays the crosssectional morphology of the prepared porous PCEC microspheres/alginate hybrid hydrogel (MPs/Alg). The hybrid hydrogel had interconnected pores of mean diameter $\sim 195$ $\mu \mathrm{m}$, and the pores were partly incorporated with the microspheres. The compressive modulus of the MPs/Alg hybrid hydrogel scaffold was investigated. The mechanical properties of the MPs improved significantly when they blended with the alginate hydrogel. The MPs/Alg hybrid hydrogel displayed an increased compressive modulus of 123.6 $\mathrm{kPa}$ on addition of PCEC microspheres, compared to that of the alginate hydrogel itself $(18.7 \mathrm{kPa})$.
2.4. Cell Morphology on PCEC Microspheres/Alginate Hydrogel. Chondrocytes have a good tolerance to the calcium alginate scaffold because of the inert alginate scaffold maintaining their morphology and phenotype as reported. ${ }^{42}$ To assess the porous PCEC microspheres and the MPs/Alg hydrogel for the cultivation of chondrocytes, articular chondrocytes were seeded and cultured on the two scaffolds for 3 days. Then, SEM was used to observe the chondrocytes grown on the scaffolds to evaluate cell morphology and proliferation. Figure 6A, B shows chondrocytes growing on PCEC microspheres and MPs/Alg hydrogel, respectively. It was found that the chondrocytes were first anchored on the superficial area of both scaffolds and kept their spherical morphology. Furthermore, the production of GAG is a critical feature of cartilage cells, which plays an important role in the phenotype regulation of the chondrocytes. As Figure 6C displays, the GAG amount of chondrocytes culture on the microspheres/alginate hydrogel increased significantly over time. This indicates that the microspheres/alginate hydrogel 

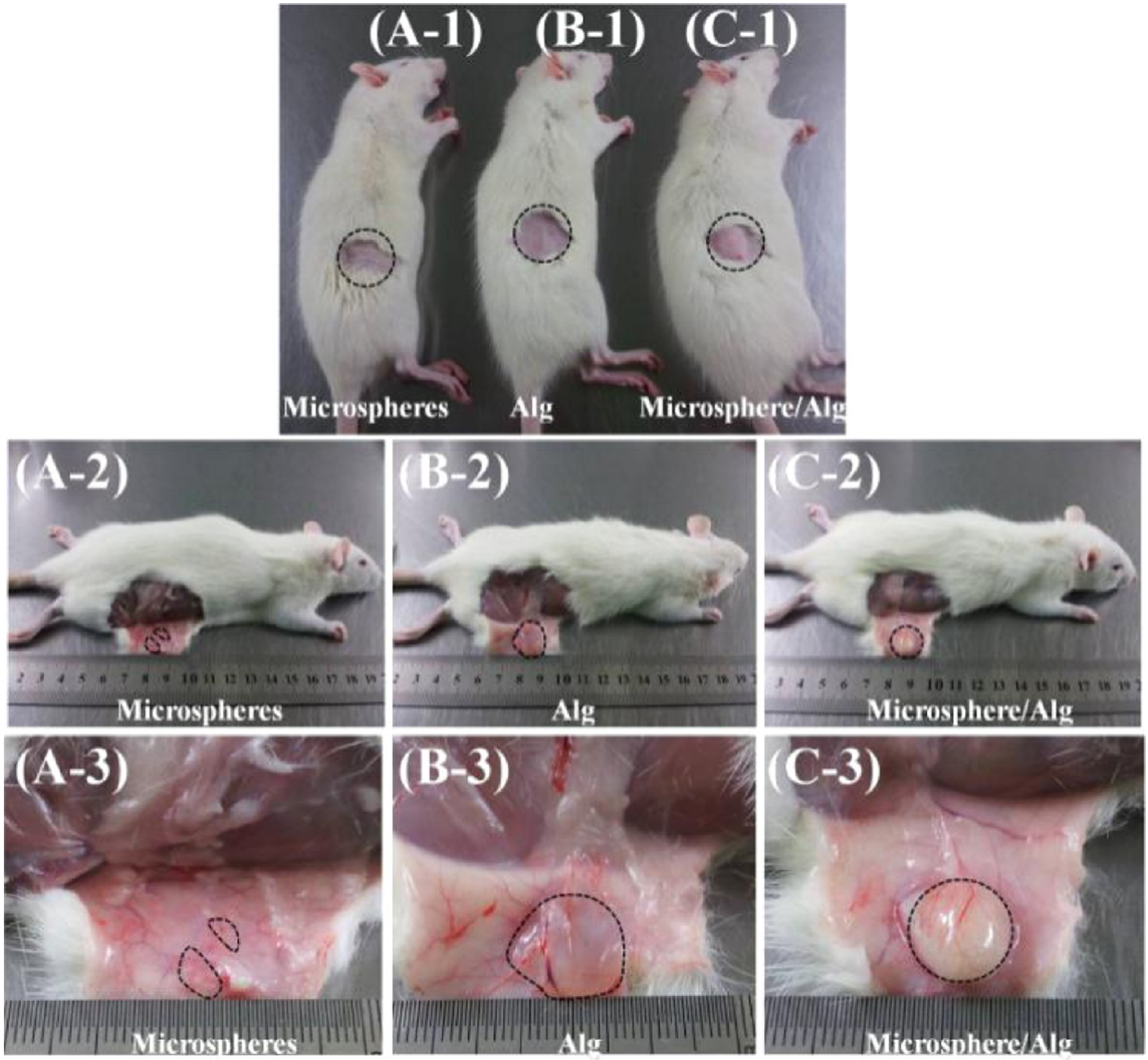

Figure 7. Fixed shapes of (A-1-A-3) porous PCEC microspheres, (B-1-B-3) 2\% alginate solution, and (C-1-C-3) porous PCEC microspheres/ alginate hydrogels after subcutaneous injection in Wistar rats.

was compatible and supplied a microenvironment, which facilitates the proliferation of chondrocytes.

2.5. In Vivo Formability and Absorption of Hybrid Scaffold. To examine the application potential of using the PCEC microspheres/alginate hydrogel for injectable therapy, the PCEC microspheres, alginate, and hybrid scaffold were implanted into mice for in vivo formability and degradability. The PCEC microspheres/alginate hydrogel could be successfully administered by injection through a syringe, instead of a surgical procedure. The scaffolds with diameter of up to $1 \mathrm{~cm}$ were collected into a suspension solution for injection. The PCEC microspheres/alginate hydrogel could pass through an $18 \mathrm{G}$ needle without eliciting a clogging problem in the needle during the injection. Tissue mounds were formed immediately after the injection (Figure 7C-1-C-3). However, the microspheres cannot be concentrated in one place and may diffuse to other sites after implantation (Figure 7A-1-A-3), which may cause inflammation and embolization. ${ }^{43}$ Also, an $1.5 \%$ alginate adhesive solution is not stable enough to fix a circle under the subcutaneous tissue (Figure 7B-1-B-3).

After implantation for 1 week, the Alg and MPs/Alg scaffold had no obvious loss (Figure S3B-1,C-1). Over time, progressive absorption of the implanted composite occurred. The materials (Alg and MPs/Alg scaffold) became relatively small at 2 weeks compared to the state at 1 week (Figure S3B-2,C-2), indicating that part of the implanted composite has been absorbed. However, the Alg group needed shorter time to be absorbed by the body, and the remaining Alg scaffold was significantly smaller compared to the hybrid scaffold at 3 weeks (Figure S3B-3,C-3). Thus, the longer time required for the degradation of the MPs/Alg scaffold was suitable for cartilage tissue engineering. Furthermore, the shape of the MPs/Alg hydrogel remained unchanged during the implantation process. However, it is difficult to observe the formability and absorption of PCEC microspheres (Figure S3A-1-A-3).

2.6. In Vivo Study of the PCEC Microspheres/Alginate Hydrogel in Rabbit Cartilage Defects. The ability of the scaffold to improve cartilage regeneration was evaluated by the reconstruction of full-thickness cartilage defect on the left knees of rabbits. The images of different treated groups are presented in Figure S4. During postoperative period, the rabbits did not show significant inflammation and rejection response. Their incised skins healed gradually, and all animals remained in good health. Then, we assessed the cartilage regenerative capability by micro-CT reconstruction evaluation, histological score analysis, and immunohistochemical assessment.

2.6.1. Micro-CT Reconstruction Evaluation. In the animal experiment, a full-thickness cartilage defect was used as the 

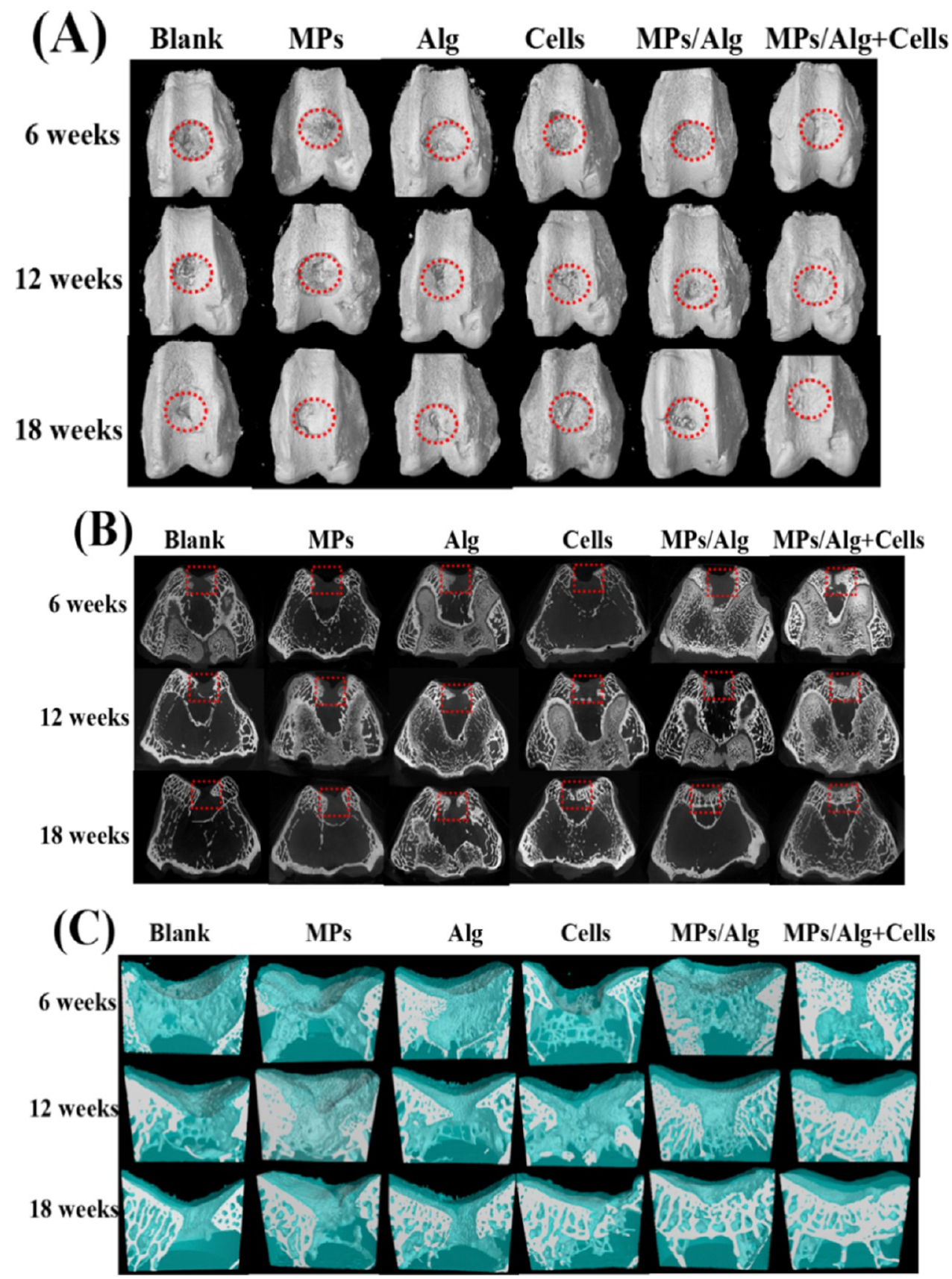

Figure 8. Micro-CT images acquired from (A) 3D reconstruction (red circles indicated the defect areas), (B) 2D reconstruction in longitudinal view (red squares indicated the defect areas), and (C) 3D reconstruction of the repaired cartilage defect of different groups (the newly grown cartilage and bone are indicated by blue and gray, respectively) after operation at different periods.

defect model, which stretched deep beneath the tidemark, without penetrating the subchondral bone plate. ${ }^{44}$ Thus, this defect model involved the cartilage and part of the subchondral bone. As shown in the 3D (Figure 8A) and two-dimensional (2D) (Figure $8 \mathrm{~B}$ ) reconstruction images, the newly regenerated tissue generally grew from the margin of defects to the central areas. After 18 postoperative weeks, the defects treated with MPs/Alg hydrogel and MPs/Alg hydrogel with cells supplement were mainly covered with a new cartilage and a subchondral bone. On the contrary, the regeneration of defects in other four groups appeared depressed, and there was still a blank zone after 18 weeks. Then, we isolated the defect areas as the region of interest (ROI) (Figure $8 \mathrm{C}$ ). The regenerated cartilage and bone were indicated by blue and gray, respectively.
It is worth noting that the defect in the MPs/Alg+ cells-treated group was almost repaired, and a regenerated matching cartilage layer was formed.

2.6.2. Semiquantitative Histological Scoring Analysis. As the repair efficacy is an important feature of defect substitutes, the regeneration percentage of bone tissue is generally marked. Because the defect sections were treated as ROI, the quantitative data about the volume of cartilage and subchondral bone could be analyzed. The percentage of newly grown bone volume in the MPs/Alg group progressed from $43.8 \%$ at 6 weeks to $89.9 \%$ at 18 weeks after implantation (Figure 9A). Moreover, $96.7 \%$ of the osteochondral tissue was regenerated by MPs/Alg+ cells at 18 weeks, which spread the largest 

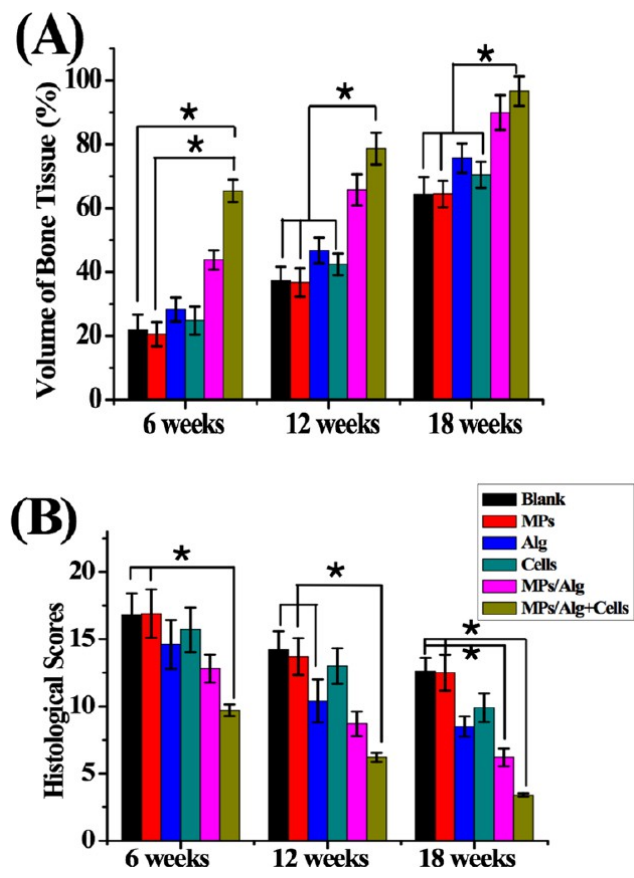

Figure 9. (A) Volume of newly formed bone tissue and (B) histological scoring for reparative tissues in different groups at 6,12 , and 18 weeks after operation. $(* p<0.05)$.

volume of the defect compared to that of blank, MPs, Alg, and cells groups.

The histological score assessment was evaluated from five aspects, as shown in Table S1. The score of the MPs/Alg+ cells group was better (i.e., lower) than that of other groups at determined time points after operation (Figure 9B); however, there was no significant difference between the MPs/Alg group and the MPs/Alg+ cells group. The results indicated that the scaffold alone played an important role in the cartilage regeneration. The structure of the scaffold had space for the growth of cartilage cells. Moreover, the characteristics of the scaffold may enhance the immigrated MSC cells to chondrogenic differentiation. Along with the cell proliferation, the scaffold degraded and was absorbed by the body, and a new cartilage was formed instead of scaffold.

2.6.3. Histological Immunohistochemical Evaluation. By $\mathrm{H} \& \mathrm{E}$ staining analysis, 6 weeks after treatment with the MPs/ Alg + cells, the newly formed cartilage cells were uniformly distributed without orderly aligning in the defects, whereas few newly formed tissues appeared in the control group (Figure 10A). A group of scaffold-treated defects could be observed in some chondrocytic cells. It is interesting that the MPs/Alg+ cells group regrew a layer of cartilage, which highly expressed GAG (Figure 10B) and COL II (Figure 10C). The group of MPs/Alg also showed the recovered hyaline cartilage and the subchondral bone. In contrast, defects in control or those transplanted with Alg, MPs, or cells only showed a limited repair, resulting in a negligible expression of GAG and COL II. Over time, a number of fibrocartilage cells appeared in control, MPs, and cell groups. The Alg-group-induced repair tissues exhibited an irregular link with the original cartilage compared to that of the MPs/Alg+ cells and MPs/Alg groups. However, the Alg-treated group was superior to the MPs and cells groups. It is worth noting that the MPs/Alg+ cells-treated group displayed even a normal regenerated subchondral bone and chondrocytes. Meanwhile, it is difficult to distinguish the interface between the repaired cartilage and the host cartilage through the homogeneous staining of COL II and GAG. The $\mathrm{MPs} / \mathrm{Alg}$ group had a relatively thinner newly regenerated cartilage, and the junction of this cartilage and the host cartilage was irregular. The MPs/Alg hydrogel with cell supply showed a successful repair in the full-thickness cartilage defect. Thus, the injectable MPs/Alg hydrogel has been demonstrated to be an effective carrier for chondrocytes and worthy of further investigation toward the desired clinical application. The MPs/Alg hydrogel will also be evaluated as a carrier for other cells to regenerate other tissues in the future.

\section{CONCLUSIONS}

In summary, we demonstrated the feasibility of calcium gluconate cross-linked alginate hydrogel prepared using biodegradable porous microsphere as the cross-linker carrier as an injectable hybrid scaffold. This injectable scaffold may be useful to meet different shape defects and regrow cartilage layers by a minimally invasive approach. The hybrid hydrogel has desirable features, such as interconnected pores, enhanced compressive modulus, good formability, and reasonable degradability. Chondrocytes seeded on the hydrogel could proliferate well and maintain their chondrogenic property. Then, the reparative ability of the porous PCEC microspheres/ alginate hydrogel was assessed in repairing full-thickness cartilage defects in a rabbit model. The results indicated that the porous PCEC microspheres/alginate hydrogel is a suitable substrate for cartilage tissue engineering.

\section{EXPERIMENTAL SECTIONS}

4.1. Materials. Poly(ethylene glycol) (PEG, $\left.M_{\mathrm{n}}=4000\right), \varepsilon$ caprolactone $(\varepsilon-\mathrm{CL})$, stannous octoate $\left(\mathrm{Sn}(\mathrm{Oct})_{2}\right)$, and poly(vinyl alcohol) (PVA, average $M_{\mathrm{n}}=30000-70000$ ) were obtained from Sigma-Aldrich Company. Calcium Dgluconate monohydrate was purchased from Aladdin Industrial Corp., Shanghai, China. Alginate, ammonium bicarbonate $\left(\mathrm{NH}_{4} \mathrm{HCO}_{3}\right)$, dichloromethane, phosphate buffer saline, and sodium hydroxide were acquired from Kelong Chemicals, Chengdu, China. Deionized water $(18.2 \mathrm{M} \Omega \mathrm{cm})$ obtained from Milli-Q Gradient System was used in all of the preparations.

For the animal experiment, we purchased 54 New Zealand White rabbits (male; initial weight: $2-2.5 \mathrm{~kg}$ ) from the Experimental Animals Center of Sichuan Province, China. They were separated into six groups evenly on the basis of the different time points. A total of 36 Wistar rats (male; weight: 200 g), purchased from Beijing HFK Bioscience Co., Ltd., were used to investigate the formability and absorption of the scaffolds in vivo. All animal studies were approved by the animal care and use committee of the State Key Laboratory of Biotherapy, Sichuan University.

4.2. Preparation of PCEC Copolymer. PCEC copolymers with different designed molecular weights were synthesized by ring-opening polymerization of poly $(\varepsilon$-caprolactone) $(\varepsilon-\mathrm{CL})$ and poly(ethylene glycol) (PEG, $\left.M_{\mathrm{n}}=4000\right)$ catalyzed by $\mathrm{Sn}(\mathrm{Oct})_{2}$ at $130{ }^{\circ} \mathrm{C}$ in accordance with our previous reports. ${ }^{45}$ The obtained PCEC copolymers were characterized by ${ }^{1} \mathrm{H}$ NMR spectroscopy (Bruker 400 spectrometer, German) and GPC (HLC-8320GPC, EcoSEC, TOSOH, Japan).

4.3. Preparation of Porous PCEC Microspheres. Porous PCEC microspheres were fabricated by a w/o/w doubleemulsion method. In brief, $3.75 \mathrm{~mL}$ of $5 \% \mathrm{NH}_{4} \mathrm{HCO}_{3}$ solution 

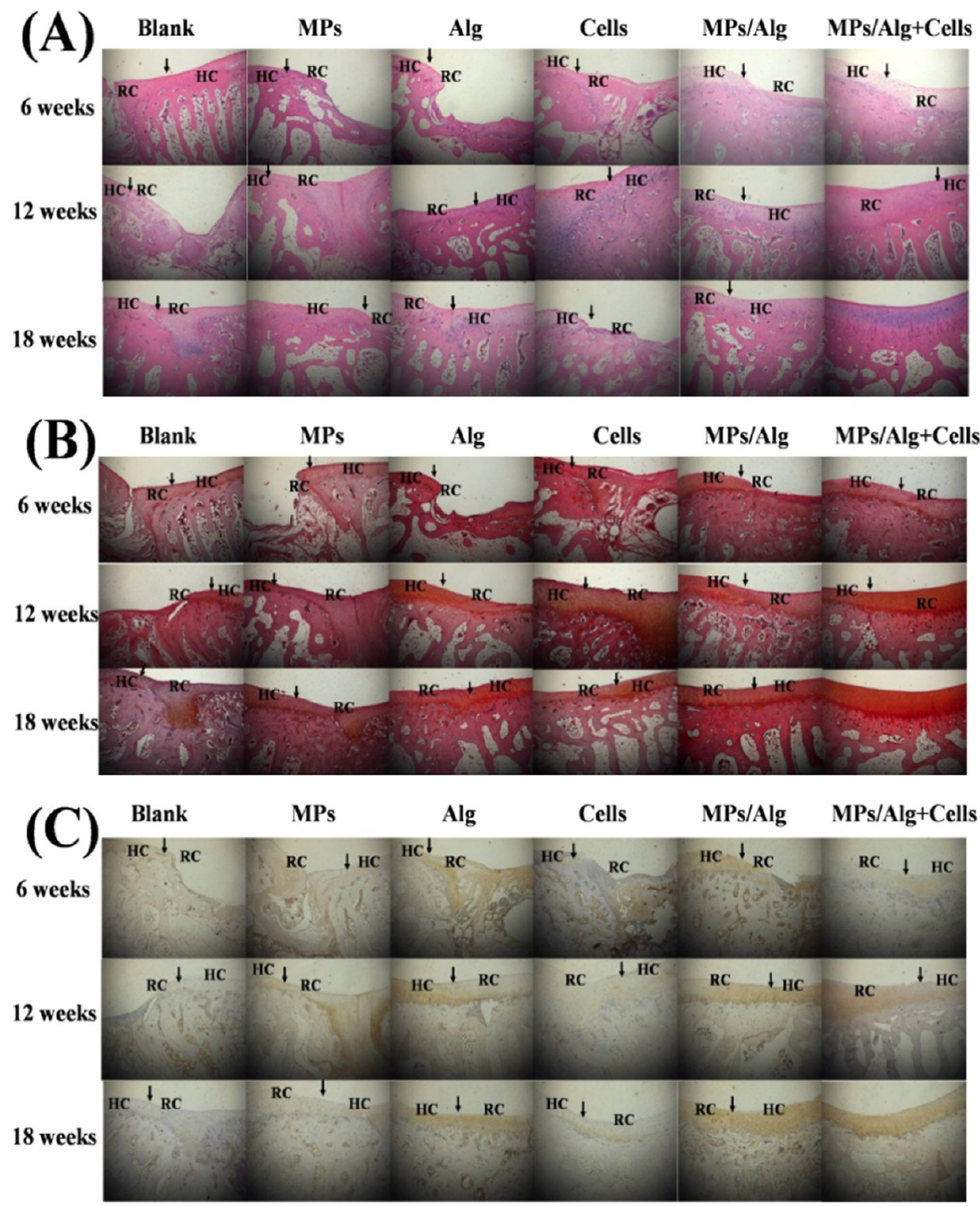

Figure 10. (A) H\&E staining, (B) Saf-O staining, and (C) COL II staining in six groups at 6, 12, and 18 weeks after operation (magnification 100X). Note: the arrows represent the host cartilage and repair cartilage boundary; RC and HC represent repaired cartilage and host cartilage, respectively.

was injected into $12 \mathrm{~mL}$ of $62.5 \%$ PCEC methylene chloride solution using a Powergen 700 homogenizer at $5000 \mathrm{rpm}$ for 3 min. Then, the first w/o emulsion was poured into $450 \mathrm{~mL}$ of $0.5 \%$ PVA solution immediately and re-emulsified using an overhead propeller (LR-400A, Fisher Scientific Co.) at 1500 rpm for $8 \mathrm{~h}$. After methylene chloride was evaporated, the obtained microspheres were separated by a filter net, etched with $\mathrm{NaOH}$, washed with distilled water, and lyophilized by a lyophilizer. The morphologies of the microspheres were observed by a digital image system (Nikon E 600 Microscope with a Nikon Digital Camera DXM 1200, Nikon Corporation, Japan) and SEM (JSM-5900LV, JEOL, Japan).

4.4. Calcium Gluconate Deposited in PCEC Microspheres. Porous PCEC microspheres were soaked in a $3 \%$ calcium gluconate solution for $3 \mathrm{~h}$. Then, the entire calcium gluconate solution was decanted, and $\mathrm{EtOH}$ was added to deposit calcium gluconate in the pores of PCEC microspheres. The above process was conducted three times. Initially, when the microspheres were soaked in $\mathrm{EtOH}$ with the reverse sequence, calcium gluconate was loaded on the surface of the microspheres. The obtained calcium gluconate-loaded microspheres were lyophilized. The morphologies of the calcium gluconate deposited in the microspheres were observed by SEM, and elemental analysis was performed by an EDX setup installed in the SEM. The amounts of calcium gluconate were measured by ICP-AES (SPECTRO ARCOS, Spectro, Germany).

4.5. Preparation and Characterizations of PCEC Microspheres/Alginate Hydrogel. First, $10 \mathrm{~mL}$ of $1.5 \%$ stock alginate solution was prepared. Then, $50 \mathrm{mg}$ of calcium Dgluconate monohydrate-loaded microspheres were mixed with the alginate stock solution $(0.75 \mathrm{~mL})$ while homogenizing the mixture to obtain a homogeneous distribution of microspheres and calcium ion cross-linking. The cross-sectional morphologies of the microspheres/alginate hydrogel were analyzed by SEM after lyophilization. The mechanical properties of the PCEC microspheres/alginate (MPs/Alg) hydrogel were measured by an Instron 5500 mechanical tester (Instron Cor.). 
4.6. Cartilage Cells Culture on the PCEC Microspheres and Hybrid Microspheres/Alg Hydrogel. Primary articular cartilage cells were obtained from the knee joints of newborn rabbits. The cartilage layers were isolated and cut into small pieces, and $0.2 \%$ collagenase II was used to digest the cartilage slices. To obtain the chondrocytes, the digested cell suspension was filtered and centrifuged. The primary chondrocytes were cultured in Dulbecco's Modified Eagle Medium with $100 \mathrm{U} /$ $\mathrm{mL}$ of penicillin, $10 \%$ fetal calf serum, and $100 \mu \mathrm{g} / \mathrm{mL}$ of streptomycin at $37{ }^{\circ} \mathrm{C}$ under $5 \% \mathrm{CO}_{2}$. When the chondrocytes were cultured to passage 3 , they were digested to grow on scaffolds; $1.0 \times 10^{4}$ chondrocytes were added into the sterilized microspheres and the hybrid hydrogel in 24-well culture plates. The cell-seeded plates were kept in an incubator for predetermined times. Then, the chondrocytes/scaffolds composites were fixed in $10 \%$ formalin solution, dehydrated in graded ethanol series, and observed by SEM. The GAG contents for different time intervals were also analyzed.

4.7. In Vivo Formability and Absorbability Investigation. The formability and biocompatibility of the microspheres/alginate hydrogel were in vivo carried out by implanting the scaffolds into the back subcutaneous tissue of Wistar rats. Cobalt-60 irradiation of $25 \mathrm{kGy}$ for $2 \mathrm{~h}$ (Sichuan Academy of Agricultural Sciences) was applied to sterilize the porous microspheres in this study. The alginate solution was filtrated by a $0.22 \mu \mathrm{m}$ filter membrane for sterilization. The microspheres, alginate solution, and their mixture were separately injected into the back subcutaneous tissue of the rats. Then, three rats in each group at each time point were sacrificed by an overdose of chloral hydrate. The residual scaffolds in the subcutaneous tissues were photographed to record the formability and absorption of scaffold and tissue appearance around the treated site.

4.8. Cartilage Defect Repair in Vivo. The cartilage defect experiment in vivo was carried out in our previous study. ${ }^{46}$ Briefly, we created full-thickness defect (thickness: $3 \mathrm{~mm}$; diameter: $4 \mathrm{~mm}$ ) through the articular cartilage and subchondral bone of the patellar groove using an electric drill. The cartilage defects in the left leg of New Zealand White rabbits were treated with nothing, microspheres, alginate hydrogel, cartilage cells, microsphere/alginate hydrogel, and microsphere/alginate hydrogel with cell supplement (each group, $n=9$ ). The animals were given antibiotic for 1 week postoperatively, housed at a constant temperature $\left(22^{\circ} \mathrm{C}\right)$, and given food and tap water. Joint samples were gathered at predetermined time points after operation and processed for micro-CT reconstruction and histological analysis. A micro-CT scanner (Y. Cheetah, YXLON International GmbH, Germany) was used to reconstruct the cartilage defect and analyze the results. The scan settings were as follows: X-ray voltage $=56$ $\mathrm{kV}, \mathrm{X}$-ray current $=61 \mu \mathrm{A}$, scaling coefficient $=50$, and voxel resolution $=0.012 \mathrm{~mm}$. The scan images were then reconstructed to create a $3 \mathrm{D}$ geometry using VGStudioMax software. For histological examination, the tissues underwent a series of processes and were stained with Hematoxylin and Eosin (H\&E), Safrannin-O (Saf-O), and Collagen type II (COL II).

\section{ASSOCIATED CONTENT}

\section{(S Supporting Information}

The Supporting Information is available free of charge on the ACS Publications website at DOI: 10.1021/acsomega.6b00495.
Characterization data, degradation ability, cartilage defect, and standard of histological score assessment (PDF).

\section{AUTHOR INFORMATION}

\section{Corresponding Author}

*E-mail: anderson-qian@163.com. Tel/Fax: +86-28-85501986.

ORCID

ZhiYong Qian: 0000-0003-2992-6424

\section{Notes}

The authors declare no competing financial interest.

\section{ACKNOWLEDGMENTS}

This work was financially supported by the National Natural Science Foundation of China (31525009 and 31271021), National High-Tech Project of China (863-Project, 2015AA020316), Sichuan Innovative Research Team Program for Young Scientists (2016TD0004), the International Science \& Technology Cooperation Program of China (2013DFG52300), and Distinguished Young Scholars of Sichuan University (2011SCU04B18). The authors thank Hui Wang (Analytical \& Testing Center, Sichuan University) for her help in SEM analysis.

\section{REFERENCES}

(1) Rothenfluh, D. A.; Bermudez, H.; O’Neil, C. P.; Hubbell, J. A. Biofunctional Polymer Nanoparticles for Intra-articular Targeting and Retention in Cartilage. Nat. Mater. 2008, 7, 248-254.

(2) Sridhar, B. V.; Brock, J. L.; Silver, J. S.; Leight, J. L.; Randolph, M. A.; Anseth, K. S. Development of a Cellularly Degradable PEG Hydrogel to Promote Articular Cartilage Extracellular Matrix Deposition. Adv. Healthcare Mater. 2015, 4, 702-713.

(3) Naranda, J.; Sušec, M.; Maver, U.; Gradišnik, L.; Gorenjak, M.; Vukasović, A.; Ivković, A.; Rupnik, M. S.; Vogrin, M.; Krajnc, P. Polyester Type PolyHIPE Scaffolds with an Interconnected Porous Structure for Cartilage Regeneration. Sci. Rep. 2016, 6, No. 28695.

(4) Fragonas, E.; Valente, M.; Pozzi-Mucell, M.; Toffanin, R.; Rizzo, R; Silvestri, F.; Vittur, F. Articular Cartilage Repair in Rabbits by Using Suspensions of Allogenic Chondrocytes in Alginate. Biomaterials 2000, 21, 795-801.

(5) Singh, Y. P.; Bhardwaj, N.; Mandal, B. B. Potential of Agarose/ Silk Fibroin Blended Hydrogel for in Vitro Cartilage Engineering. ACS Appl. Mater. Interfaces 2016, 8, 21236-21249.

(6) Pelttari, K.; Pippenger, B.; Mumme, M.; Feliciano, S.; Scotti, C.; Mainil-Varlet, P.; Procino, A.; Rechenberg, B.; Schwamborn, T.; Jakob, M.; Cillo, C.; Barbero, A.; Martin, I. Adult Human Neural Crestderived Cells for Articular Cartilage Repair. Sci. Transl. Med. 2014, 6, No. 251 ral19.

(7) Chen, W.; Chen, S.; Morsi, Y.; El-Hamshary, H.; El-Newhy, M.; Fan, C.; Mo, X. Superabsorbent 3D Scaffold Based on Electrospun Nanofibers for Cartilage Tissue Engineering. ACS Appl. Mater. Interfaces 2016, 8, 24415-24425.

(8) Armstrong, J. P. K.; Shakur, R.; Horne, J. P.; Dickinson, S. C.; Armstrong, C. T.; Lau, K.; Kadiwala, J.; Lowe, R.; Seddon, A.; Mann, S.; Anderson, J. L. R.; Perriman, A. W.; Hollande, A. P. Artificial Membrane-Binding Proteins Stimulate Oxygenation of Stem Cells During Engineering of Large Cartilage Tissue. Nat. Commun. 2015, 6, No. 7405.

(9) Lee, J. E.; Kim, K. E.; Kwon, I. C.; Ahn, H. J.; Lee, S.-H.; Cho, H.; Kim, H. J.; Seong, S. C.; Lee, M. C. Effects of the Controlled-Released TGF- $\beta 1$ from Chitosan Microspheres on Chondrocytes Cultured in a Collagen/Chitosan/Glycosaminoglycan Scaffolde. Biomaterials 2004, $25,4163-4173$. 
(10) Zhang, J.; Wang, J. H.-C. Kartogenin Induces Cartilage-like Tissue Formation in Tendon-bone Junction. Bone Res. 2014, 2, No. 14008.

(11) Giammanco, G. E.; Carrion, B.; Coleman, R. M.; Ostrowski, A. D. Photoresponsive Polysaccharide-Based Hydrogels with Tunable Mechanical Properties for Cartilage Tissue Engineering. ACS Appl. Mater. Interfaces 2016, 8, 14423-14429.

(12) Joeng, K. S.; Long, F. Wnt7b can Replace Ihh to Induce Hypertrophic Cartilage Vascularization but not Osteoblast Differentiation during Endochondral Bone Development. Bone Res. 2014, 2, No. 14004.

(13) Zhang, N.; Lock, J.; Sallee, A.; Liu, H. Magnetic Nanocomposite Hydrogel for Potential Cartilage Tissue Engineering: Synthesis, Characterization, and Cytocompatibility with Bone Marrow Derived Mesenchymal Stem Cells. ACS Appl. Mater. Interfaces 2015, 7, 2098720998.

(14) Thoniyot, P.; Tan, M. J.; Karim, A. A.; Young, D. J.; Loh, X. J. Nanoparticle-Hydrogel Composites: Concept, Design, and Applications of These Promising, Multi-Functional Materials. Adv. Sci. 2015, 2, No. 1400010.

(15) Ren, K.; He, C.; Xiao, C.; Li, G.; Chen, X. Injectable Glycopolypeptide Hydrogels as Biomimetic Scaffolds for Cartilage Tissue Engineering. Biomaterials 2015, 51, 238-249.

(16) Elisseeff, J. Injectable Cartilage Tissue Engineering. Expert Opin. Biol. Ther. 2004, 4, 1849-1859.

(17) Khetan, S.; Guvendiren, M.; Legant, W. R.; Cohen, D. M.; Chen, C. S.; Burdick, J. A. Degradation-Mediated Cellular Traction Directs Stem Cell Fate in Covalently Crosslinked Three-Dimensional Hydrogels. Nat. Mater. 2013, 12, 458-465.

(18) Söntjens, S. H.; Nettles, D. L.; Carnahan, M. A.; Setton, L. A.; Grinstaff, M. W. Biodendrimer-based Hydrogel Scaffolds for Cartilage Tissue Repair. Biomacromolecules 2006, 7, 310-316.

(19) Kim, K. S.; Park, S. J.; Yang, J. A.; Jeon, J.-H.; Bhang, S. H.; Kim, B. S.; Hahn, S. K. Injectable Hyaluronic Acid-tyramine Hydrogels for the Treatment of Rheumatoid Arthritis. Acta Biomater. 2011, 7, 666674.

(20) Chen, F.; Yu, S.; Liu, B.; Ni, Y.; Yu, C.; Su, Y.; Zhu, X.; Yu, X.; Zhou, Y.; Yan, D. An Injectable Enzymatically Crosslinked Carboxymethylated Pullulan/Condroitin Sulfate Hydrogel for Cartilage Tissue Engineering. Sci. Rep. 2016, 6, No. 20014.

(21) Vickers, S. M.; Gotterbarm, T.; Spector, M. Cross-Linking Affects Cellular Condensation and Chondrogenesis in Type II Collagen-GAG Scaffolds Seeded with Bone Marrow-Derived Mesenchymal Stem Cells. J. Orthop. Res. 2010, 28, 1184.

(22) Lee, C. R.; Grodzinsky, A. J.; Spector, M. The Effects of CrossLinking of Collagen-Glycosaminoglycan Scaffolds on Compressive Stiffness, Chondrocyte-Mediated Contraction, Proliferation and Biosynthesis. Biomaterials 2001, 22, 3145-3154.

(23) Ko, C. S.; Huang, J. P.; Huang, C. W.; Chu, I. M. Type II Collagen-Chondroitin Sulfate-Hyaluronan Scaffold Cross-Linked by Genipin for Cartilage Tissue Engineering. J. Biosci. Bioeng. 2009, 107, $177-182$.

(24) Wieland, J. A.; Houchin-Ray, T. L.; Shea, L. D. Non-Viral Vector Delivery from PEG-Hyaluronic Acid Hydrogels. J. Controlled Release 2007, 120, 233-241.

(25) Ferretti, M.; Marra, K. G.; Kobayashi, K.; Defail, A. J.; Chu, C. R. Controlled in vivo Degradation of Genipin Crosslinked Polyethylene Glycol Hydrogels within Osteochondral Defects. Tissue Eng. 2006, 12, 2657.

(26) Kehr, N. S.; Prasetyanto, E. A.; Benson, K.; Ergün, B.; Galstyan, A.; Galla, H.-J. Periodic Mesoporous Organosilica-Based Nanocomposite Hydrogels as Three-Dimensional Scaffolds. Angew. Chem., Int. Ed. 2013, 52, 1156-1160.

(27) Andrejecsk, J. W.; Cui, J.; Chang, W. G.; Devalliere, J.; Pober, J. S.; Saltzman, W. M. Paracrine Exchanges of Molecular Signals Between Alginate-Encapsulated Pericytes and Freely Suspended Endothelial Cells within a 3D Protein Gel. Biomaterials 2013, 34, 8899-8908.

(28) Markstedt, K.; Mantas, A.; Tournier, I.; Ávila, H. M.; Hägg, D.; Gatenholm, P. 3D Bioprinting Human Chondrocytes with Nano-
cellulose-Alginate Bioink for Cartilage Tissure Engineering Application. Biomacromolecules 2015, 16, 1489-1496.

(29) Chung, H. J.; Kim, I. K.; Kim, T. G.; Gwan, T. Park Highly Open Porous Biodegradable Microcarriers: in vitro Cultivation of Chondrocytes for Injectable Delivery. Tissue Eng., Part A 2008, 14, 607-615.

(30) Holland, T. A.; Tabata, Y.; Mikos, A. G. Dual Growth Factor Delivery from Degradable Oligo(Poly(ethyleneglycol) Fumarate) Hydrogel Scaffolds for Cartilage Tissue Engineering. J. Controlled Release 2005, 101, 111-125.

(31) Kim, H. K.; Chung, H. J.; Park, T. G. Biodegradable Polymeric Microspheres with "Open/Closed" Pores for Sustained Release of Human Growth Hormone. J. Controlled Release 2006, 112, 167-174.

(32) Kim, I.; Byeon, H. J.; Kim, T. H.; Lee, E. S.; Oh, K. T.; Shin, B. S.; Lee, K. C.; Youn, Y. S. Doxorubicin-Loaded Highly Porous Large PLGA Microparticles as a Sustained-Release Inhalation System for the Treatment of Metastatic Lung Cancer. Biomaterials 2012, 33, 55745583.

(33) Nukavarapu, S. P.; Kumbar, S. G.; Brown, J. L.; Krogman, N. R.; Weikel, A. L.; Hindenlang, M. D.; Nair, L. S.; Allcock, H. R.; Laurencin, C. T. Polyphosphazene/Nano-Hydroxyapatite Composite Microsphere Scaffolds for Bone Tissue Engineering. Biomacromolecules 2008, 9, 1818-1825.

(34) Zhang, Z.; Gupte, M. J.; Jin, X.; Ma, P. X. Injectable Peptide Decorated Functional Nanofibrous Hollow Microspheres to Direct Stem Cell Differentiation and Tissue Regeneration. Adv. Funct. Mater. 2015, 25, 350-360.

(35) Kim, T. K.; Yoon, J. J.; Lee, D. S.; Park, T. G. Gas Foamed Open Porous Biodegradable Polymeric Microspheres. Biomaterials 2006, 27, $152-159$.

(36) Lee, J.; Oh, Y. J.; Lee, S. K.; Lee, K. Y. Facile Control of Porous Structures of Polymer Microspheres Using an Osmotic Agent for Pulmonary Delivery. J. Controlled Release 2010, 146, 61-67.

(37) Fan, M.; Liao, J.; Guo, G.; Ding, Q.; Yang, Y.; Luo, F.; Qian, Z. Dexamethasone-Loaded Poly(D, L-lactic acid) Microspheres/Poly(ethylene glycol)-Poly( $\varepsilon$-caprolactone)-Poly(ethylene glycol) Micelles Composite for Skin Augmentation. J. Biomed. Nanotechnol. 2014, 10, 592-602.

(38) Lee, C. H.; Rodeo, S. A.; Fortier, L. A.; Lu, C.; Erisken, C.; Mao, J. J. Protein-Releasing Polymeric Scaffolds Induce Fibrochondrocytic Differentiation of Endogenous Cells for Knee Meniscus Regeneration in Sheep. Sci. Transl. Med. 2014, 6, No. 266ra171.

(39) Liao, J.; Shi, K.; Ding, Q.; Qu, Y.; Luo, F.; Qian, Z. Recent Developments in Scaffold-Guided Cartilage Tissue Regeneration. J. Biomed. Nanotechnol. 2014, 10, 3085-3104.

(40) Onoe, H.; Okitsu, T.; Itou, A.; Kato-Negishi, M.; Gojo, R.; Kiriya, D.; Sato, K.; Miura, S.; Iwanaga, S.; Kuribayashi-Shigetomi, K.; Matsunaga, Y. T.; Shimoyama, Y.; Takeuchi, S. Metre-Long CellLaden Microfibres Exhibit Tissue Morphologies and Functions. Nat. Mater. 2013, 12, 584-590.

(41) Wang, P.; Zhao, L.; Liu, J.; Weir, M. D.; Zhou, X.; Xu, H. H. K. Bone Tissue Engineering via Nanostructured Calcium Phosphate Biomaterials and Stem Cells. Bone Res. 2014, 2, No. 14017.

(42) Cogan, F.; Booth, A.; Gough, J. E.; Webb, S. J. Conversion of Magnetic Impulses into Cellular Responses by Self-Assembled Nanoparticle-Vesicle Hydrogels. Angew. Chem., Int. Ed. 2011, 50, 12290-12293.

(43) Morhenn, V. B.; Lemperle, G.; Gallo, R. L. Phagocytosis of Different Particulate Dermal Filler Substances by Human Macrophages and Skin Cells. Dermatol. Surg. 2002, 28, 484-490.

(44) Altman, R. D.; Kates, J.; Chun, L. E.; Dean, D. D.; Eyre, D. Preliminary Observations of Chondral Abrasion in a Canine Model. Ann. Rheum. Dis. 1992, 51, 1056-1062.

(45) Dai, M.; Xu, X.; Song, J.; Fu, S. Z.; Gou, M. L.; Luo, F.; Qian, Z. Y. Preparation of Camptothecin-Loaded PCEC Microspheres for the Treatment of Colorectal Peritoneal Carcinomatosis and Tumor Growth in Mice. Cancer Lett. 2011, 312, 189-196. 
(46) Liao, J. F.; Qu, Y.; Chu, B. Y.; Zhang, X. N.; Qian, Z. Y. Biodegradable CSMA/PECA/Graphene Porous Hybrid Scaffold for Cartilage Tissue Engineering. Sci. Rep. 2015, 5, No. 09879. 